\title{
Superparamagnetic Nanoparticles: A Biodistribution Study Using Xenopus laevis Embryos
}

\author{
Martina Giannaccini ${ }^{1,2, *}$, Alfred Cuschieri', Luciana Dente ${ }^{1}$ and Vittoria Raffa ${ }^{1,2, *}$ \\ ${ }^{1}$ Unit of Cell and Developmental Biology, Department of Biology, University of Pisa, S.S. 12 Abetone e \\ Brennero, 4, 56127 Pisa, Italy \\ ${ }^{2}$ Institute of Life Science, Scuola Superiore Sant'Anna, Piazza Martiri della Libertà 33, 56127 Pisa, Italy
}

\begin{abstract}
Various in-vivo biological models have been proposed for studying the interactions of nanomaterials with biological systems. Recently, there has been a significant increase in interest in the use of non-mammalian embryos, such as the frog Xenopus laevis as valid models for research in nanomedicine. In the present work, we demonstrate that $X$. laevis is a powerful model for the study of the biodistribution of superparamagnetic nanoparticles (SPION), extensively used in biomedical field for cell separation, MRI diagnostics and magnetic drug-targeting.

$10 \mathrm{nl}$ of $25 \mathrm{mg} / \mathrm{ml}$ of SPIONs (nano-screen MAG/ARA $200 \mathrm{~nm}$, Chemicell) were microinjected. The biodistribution of SPIONs, following cardiac or pronephros injection of anesthetized frog larvae at stage 37, was studied by both in-vivo florescence and by Prussian blue staining of paraffin sections of the larvae after $24,48,72$ or 96 hours (at $14{ }^{\circ} \mathrm{C}$ ). The study confirmed that SPIONs diffused from either injection site by blood stream to all larval organs, being still present after 96 hours of injection.
\end{abstract}

Keywords: Xenopus laevis, embryo, superparamagnetic nanoparticle, biodistribution.

\section{INTRODUCTION}

Various in-vivo biological models have been proposed for studying the interactions of nanomaterials (NMs) with biological systems. Although in widespread use, small animal models (rodents) are costly and labour intensive and furthermore raise important ethical issues and have generated resistance to life science research from the anti-vivisectionist lobby. Such issues and concerns can been allayed by using nonmammalian embryos for in-vivo studies to probe the interactions between nanomaterials and tissues.

Developmental biology offers powerful models for the study of the cell biological interactions with nanomaterials as embryos are particularly sensitive indicators of adverse biological effects on the organisms. Moreover, they provide a useful platform to define the mechanism of action of adverse effects resulting from exposure to NMs [1, 2]. Since highly coordinated cell-to-cell communications and molecular signalling are required for normal development, any perturbations by nanomaterials will disrupt orderly embryogenesis leading to abnormal development manifested as morphological malformations, behavioural changes and even embryo death.

Xenopus laevis is one of the principal animal model systems used in developmental biology since 1950 and

*Address correspondence to this author at the Unit of Cell and Developmental Biology, Department of Biology, University of Pisa, S.S. 12 Abetone e Brennero, 4, 56127 Pisa, Italy; Tel: +39-050-2211487; Fax: + 39-050-2211495; E-mail: vraffa@biologia.unipi.it, m.giannaccini@sssup.it

E-ISSN: 2311-8792 much of our knowledge about the mechanisms of vertebrate early development comes from these studies. As an experimental test system, Xenopus laevis offers several advantages: large numbers of embryos with each fecundation (thousands) with a very short early development time (3 days to reach tadpole), external development, close homology with human genes, aside from requiring much less material than small mammals for the assessment of nanomaterialbiological interactions and toxicity and less expensive husbandry/housing. Additionally, their quite large size (from $1-1.2 \mathrm{~mm}$ (zygote) to $1 \mathrm{~cm}$ (120 hour post fertilization)) facilitates manipulation and microsurgery [3].

In the present work, we investigate the use of $X$. laevis as model for biodistribution studies of magnetic nanoparticles (MNPs) as their use for various biomedical applications has increased significantly over the last decade [4]. MNPs are composed of magnetic materials such as iron, nickel, cobalt and their oxides like magnetite $\left(\mathrm{Fe}_{3} \mathrm{O}_{4}\right)$, maghemite $\left(\mathrm{\gamma}-\mathrm{Fe}_{2} \mathrm{O}_{3}\right)$, cobalt ferrite $\left(\mathrm{Fe}_{2} \mathrm{CoO}_{4}\right)$ or chromium di-oxide $\left(\mathrm{CrO}_{2}\right)$ [5]. Superparamagnetic nanoparticles (SPIONs) belong to the class of MNPs having an iron oxide core coated by either inorganic materials (silica, gold) and organic materials (phospholipids, fatty acids, polysaccharides, peptides or other surfactants and polymers). They are the most commonly used in biomedicine because of their high saturation magnetization $\mathrm{M}_{\mathrm{S}}\left(85-95 \mathrm{Am}^{2} / \mathrm{kg}\right)$, biological compatibility, environmental stability, and low synthesis costs [6]. SPIONs are useful for biological application for two reasons: their superparamagnetic ๑) 2013 Savvy Science Publisher 
nature prevents particle agglomeration [7], and because the iron is a naturally occurring metal in the human body (ferritin), SPIONs are biocompatible as the body is able to reduce the particles into their elements enabling entry in normal iron metabolism [8, 9]. Currently, many clinical diagnostic/therapeutic tools use SPIONs, e.g., MRI contrast agents in magnetic resonance imaging [10], vectors for drug delivery [11, 12], magnetic cell separation [13], gene delivery [14], etc. There are also on-going clinical trials to test the efficacy of SPIONs as contrast agent for hyperthermic ablation of recurrent glioblastoma multiforme [15]. There is a growing interest in the synthesis of new generation SPIONs with tailored features, i.e., small size, capability of surface functionalization, minimal cytotoxicity, stability, high magnetic response and ability to stably magnetize cellular structures. These newer diagnostic and treatment modalities of SPIONs underpin the increased need to characterize their interaction with biological models. In this communication, we report the potential of $X$. laevis as an efficient model for biodistribution studies of nanomaterials, using SPIONs as an exemplar.

\section{MATERIALS AND METHODS}

\section{Nanoparticles}

SPIONs used in this work are nano-screenMAGARA supplied by Chemicell. The vial, as provided by the supplier, contains an aqueous dispersion of magnetic fluorescent nanoparticles $25 \mathrm{mg} / \mathrm{ml}$. The particles have a magnetic core of magnetite and a polysaccharide matrix of glucuronic acid, a derivate of glucose. The size (hydrodynamic diameter) is about $200 \mathrm{~nm}$. The magnetite core overlaid with a red fluorescence dye (excitation: $578 \mathrm{~nm}$; emission: 613 $\mathrm{nm})$.

\section{Animal Preparation}

Animal procedures were performed in strict compliance with protocols approved by Italian Ministry of Public Health and of the local Ethical Committee of University of Pisa (authorization n. 99/2012-A, 19.04.2012), in conformity with the European Communities Council Directive of 24 November 1986 (86/609/EEC). Xenopus laevis embryos were generated and staged as described $[3,16]$. The larvae were reared at $14^{\circ} \mathrm{C}$ in MMR solution [17].

\section{Embryos Microinjections}

$10 \mathrm{nl}$ of $25 \mathrm{mg} / \mathrm{ml}$ of SPIONs were microinjected in larvae of Xenopus laevis at stages 37 (according to Nieuwkoop and Faber [3]) in the pericardial cavity or in one pronephros by a glass microneedle mounted in a Nanodrummond microinjector (Figure 1). Prior to injection larvae were immersed in $0.05-0.1 \%$ tricaine (methanesulphonate MS222, Sigma).

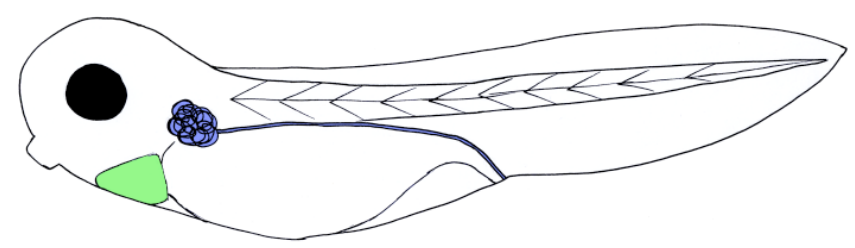

Figure 1: Graphical representation of a $X$. laevis larva, with heart (green) and pronephros (blue) in evidence.

\section{Fluorescent Analysis}

24, 48, 72, 96 hours (at $14^{\circ} \mathrm{C}$ ) after injection, fluorescence of SPIONs was detected in whole mount embryos by an epifluorescent stereomicroscope (Nikon SMZ150).

\section{Histochemical Analysis}

72 hours after microinjection embryos were formalin fixed for 1 hour, after which they were embedded in paraffin and sectioned $(14 \mu \mathrm{m})$. The sections were stained by Prussian Blue according to the manufacturer's instructions (Perls Microstain, DiaPath, Martinengo, Italy).

\section{RESULTS}

\section{Intracardiac Injection}

$250 \mathrm{ng}$ of SPIONs were injected peri/intracardiacally in 25 Xenopus larvae at stage 37 and tracked by florescence microscopy in time course experiments of whole embryos. When injected, SPIONs were observed to co-localize with the physiological movement of the hearth beats. Throughout the duration of the experiments, clusters of SPIONs remained stuck at the heart and pericardial sac edges (100\%) (Figure 2). By 48 hours the SPIONs had spread to pronephros and gut $(70 \%)$ probably by blood borne transportation (Figure 2B) to reach the final destination by 96 hours (Figure 2B-D).

\section{Left Pronephros Injection}

30 larvae at stage 37 were injected into the left pronephros. The pronephros is the precursor form of the vertebrate kidney and is the functional embryonic kidney of frogs. It consists of a single large glomus (a capillary network), a nephrocoelom (a collecting cavity), one set of tubules and a single excreting duct. Within 


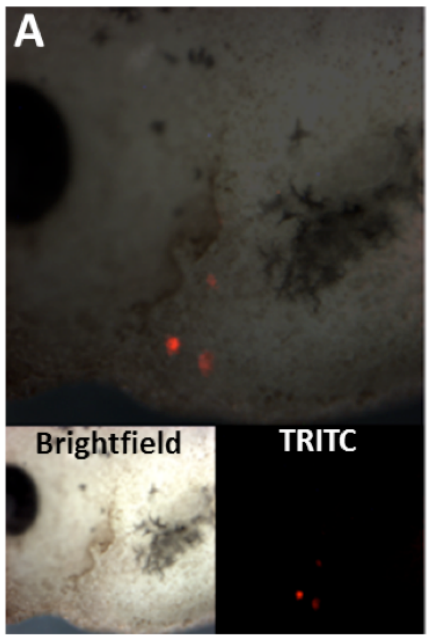

24 hours

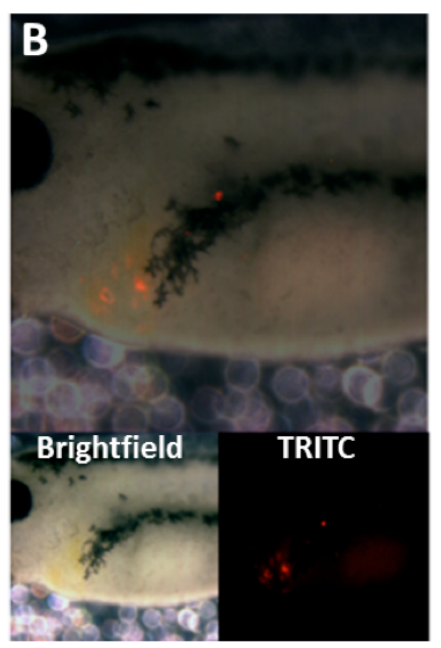

48 hours

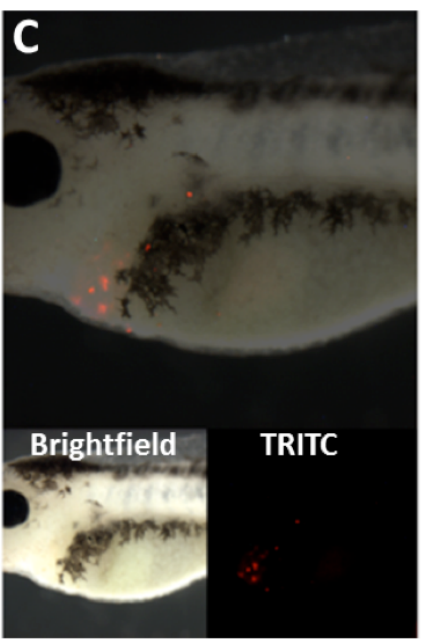

72 hours

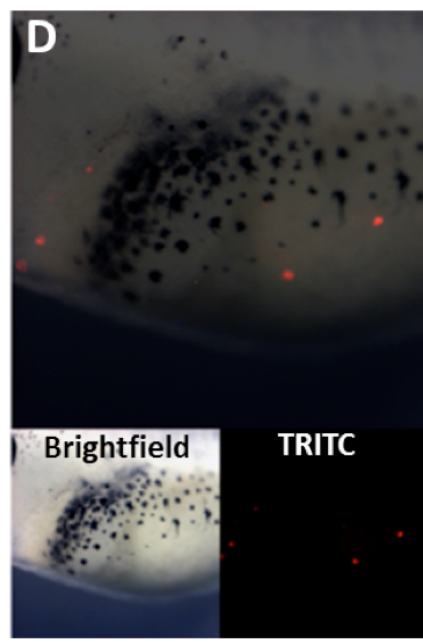

96 hours

Figure 2: Larvae injected peri/intracardiacally at stage 37: fluorescence analysis at 24, 48, 72 and 96 hours after injection (A, B, $\mathbf{C}$ and $\mathbf{D}$, respectively). Larvae are oriented with dorsal to the top and head to the left. SPIONs are red fluorescent spots.

24 hours, SPIONs remained restricted to the injected side and, specifically, to the pronephros, with a weak signal in the gut $(60 \%)$ and heart $(30 \%)$ (Figure $\mathbf{3 A}$, top). At this stage there were no SPIONs in the noninjected side (100\%) (Figure 3A, down). In contrast, at 48 hours SPIONs had spread to the entire embryos, including the non-injected side (100\%), suggesting spread by blood borne transportation (Figure 3B) reaching a peak of vessels diffusion (in some cases visible under the microscope) at 72 hours. At 96 hours, particles remained in the reached position (Figure $3 \mathbf{C}$ -
D). On the injected side we observed SPIONs in gut $(90 \%)$, heart $(90 \%)$, eye $(50 \%)$, head $(65 \%)$ (Figure 3C-D, top) and tail fin (40\%) (Figure 4); whereas in non-injected side, the distribution was pronephros $(100 \%)$, gut $(90 \%)$ and head (10\%) (Figure 3C-D, down).

In order to obtain more detailed information on the distribution of SPIONs, 10 larvae for each type of injection were subjected to histochemical analysis. Larvae were fixed 72 hours after the injection. Slices 14

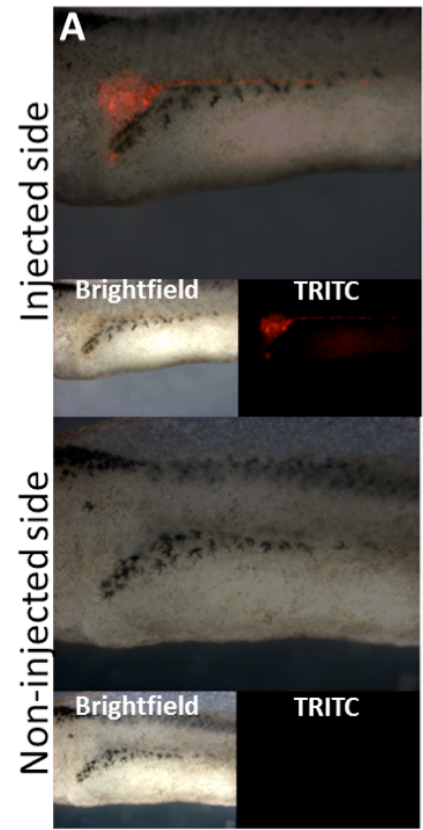

24 hours

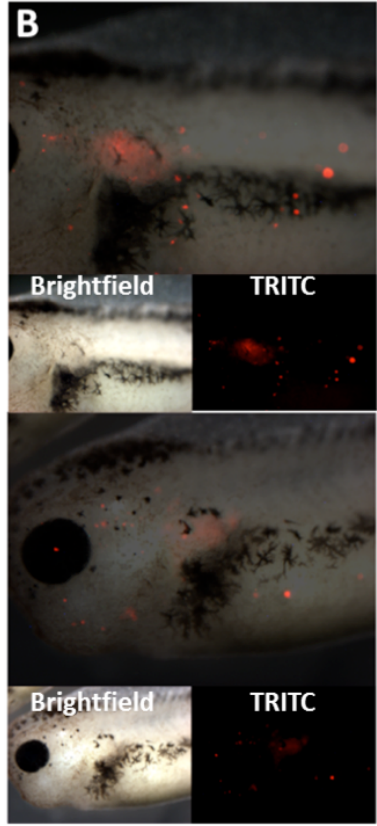

48 hours

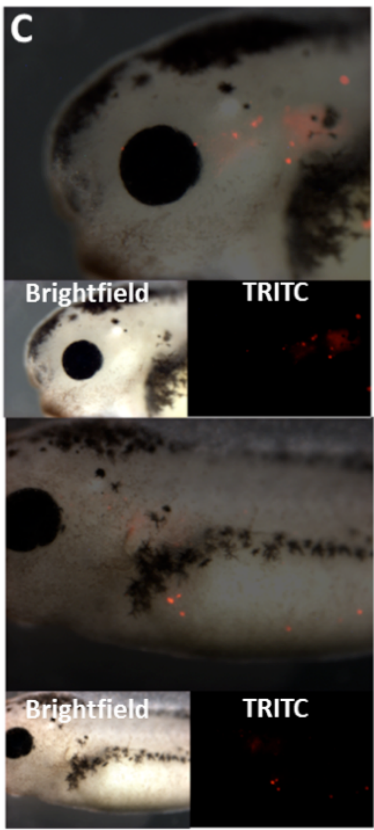

72 hours

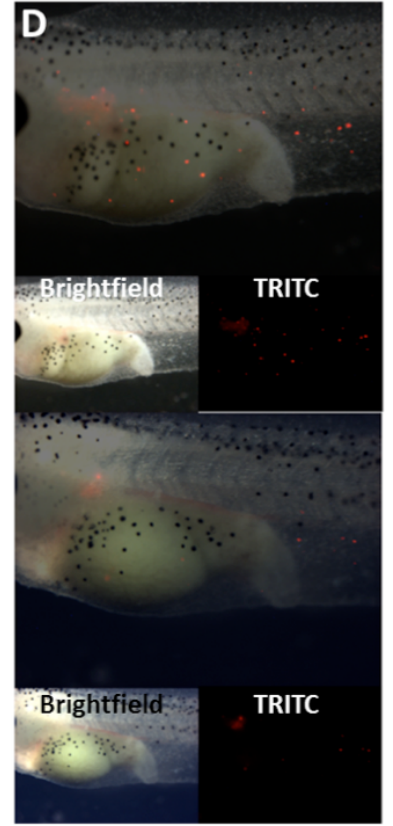

96 hours

Figure 3: Larvae injected in pronephros at stage 37: fluorescence analysis at 24, 48, 72 and 96 hours after injection (A, B, C and $\mathbf{D}$, respectively). Larvae are oriented with dorsal to the top and head to the left. Top pictures: injected side; down pictures: control non-injected side (down). SPIONs are the red fluorescent spots. 


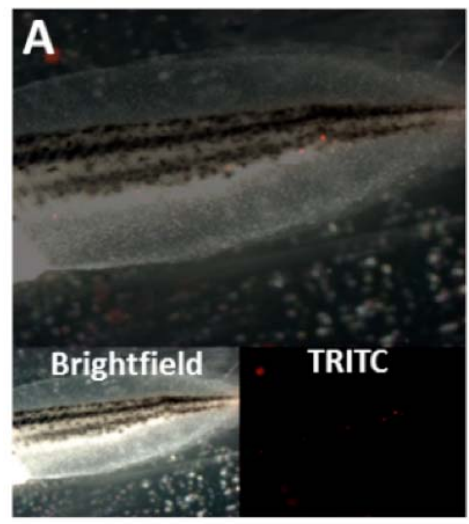

72 hours

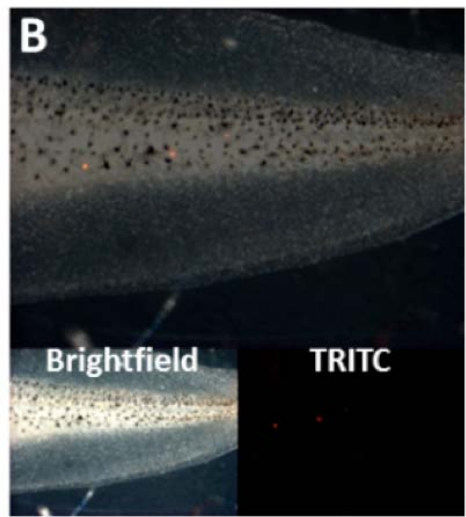

96 hours

Figure 4: Larvae injected in pronephros at stage 37: fluorescence analysis of tails at 72 and 96 hours after injection (A and B, respectively). Larvae are oriented with dorsal to the top and head to the left. SPIONs are the red fluorescent spots.

$\mu \mathrm{m}$ thick were obtained and stained with Prussian Blue kit. With this staining, iron appears blue, nuclei appear red, and cytoplasm appears pink. All slides were examined thoroughly for evidence of magnetic particles. In agreement with fluorescent analysis, the peri/intracardiacally injected larvae had SPIONs inside the heart $(40 \%)$ (Figure 5A-B) and pericardial sac (90\%) (Figure 5C), pronephros (both in glomus and tubules portion (Figure 5D-E)) (100\%) and intestine and/or peritoneum (90\%) (Figure 5F). Furthermore we observed SPIONs in the optic nerve (70\%) (Figure 5G), parachordal cartilage $(70 \%)$ (Figure $5 \mathrm{H}$ ), liver $(50 \%)$ (Figure $5 \mathrm{I}$ ), rarely in pharyngeal walls $(30 \%)$ (Figure $\mathbf{5 L})$, with low levels in the outer margin of the anterior central nervous system (CNS) (80\%) (Figure 5M) and somites (50\%) (Figure $5 \mathrm{~N}$ ).

Similar distribution was observed for larvae injected in the left pronephros. Indeed we recorded iron staining
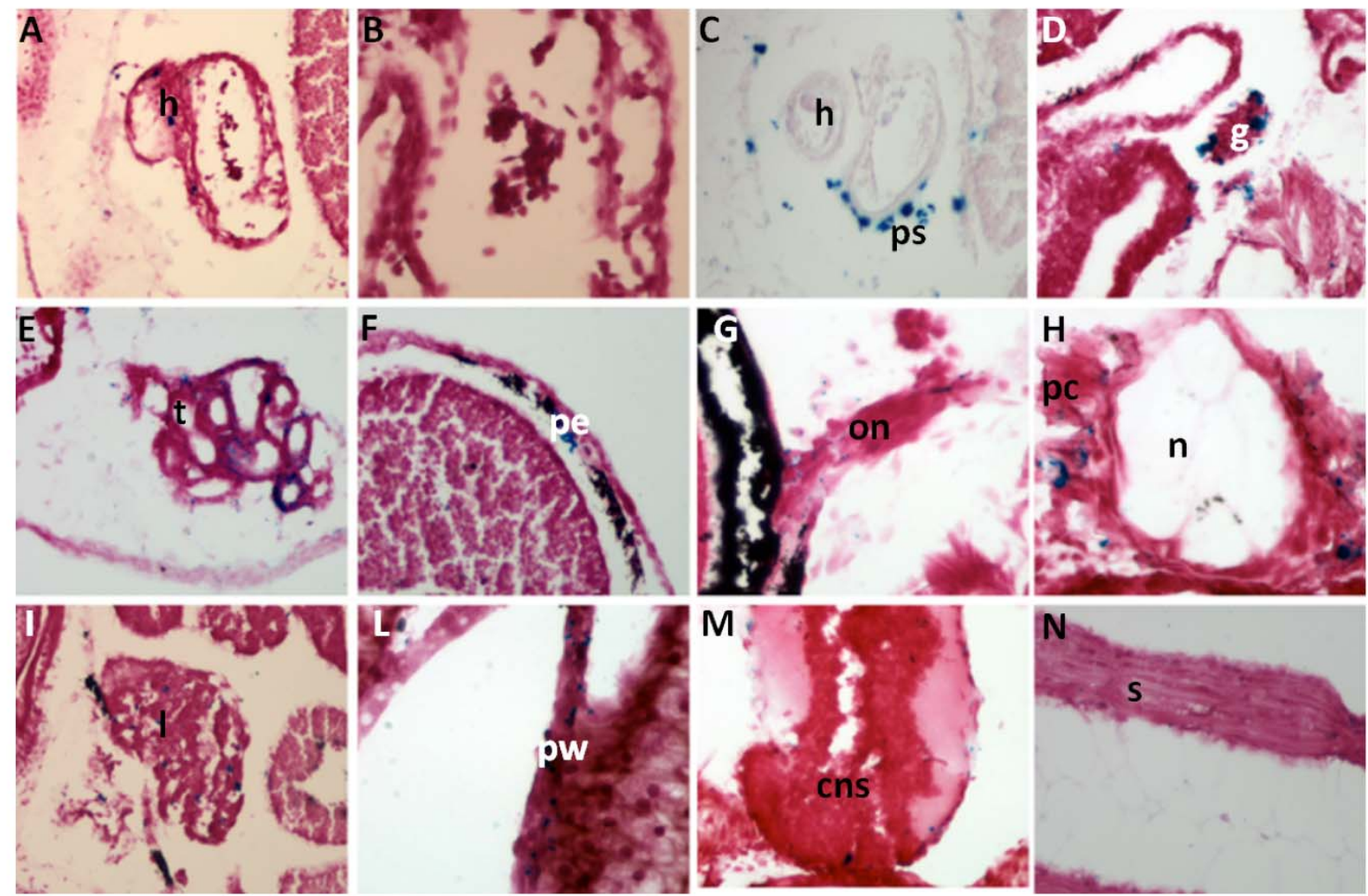

Figure 5: Iron staining of peri/intracardiacally injected larvae sections (larvae fixed 72 hours after injection). h: heart; ps: pericardial sac; g: glomus; t: tubules; pe: peritoneum; on: optic nerve; pc: parachordal cartilage; n: notochord; l: liver; pw: pharyngeal walls; cns: central nervous system; s: somites. The SPIONs are blue labeled, the sections are stained with pararosaniline and the black regions in $\mathrm{F}, \mathrm{G}$ and I are due to the natural larvae pigmentation. 


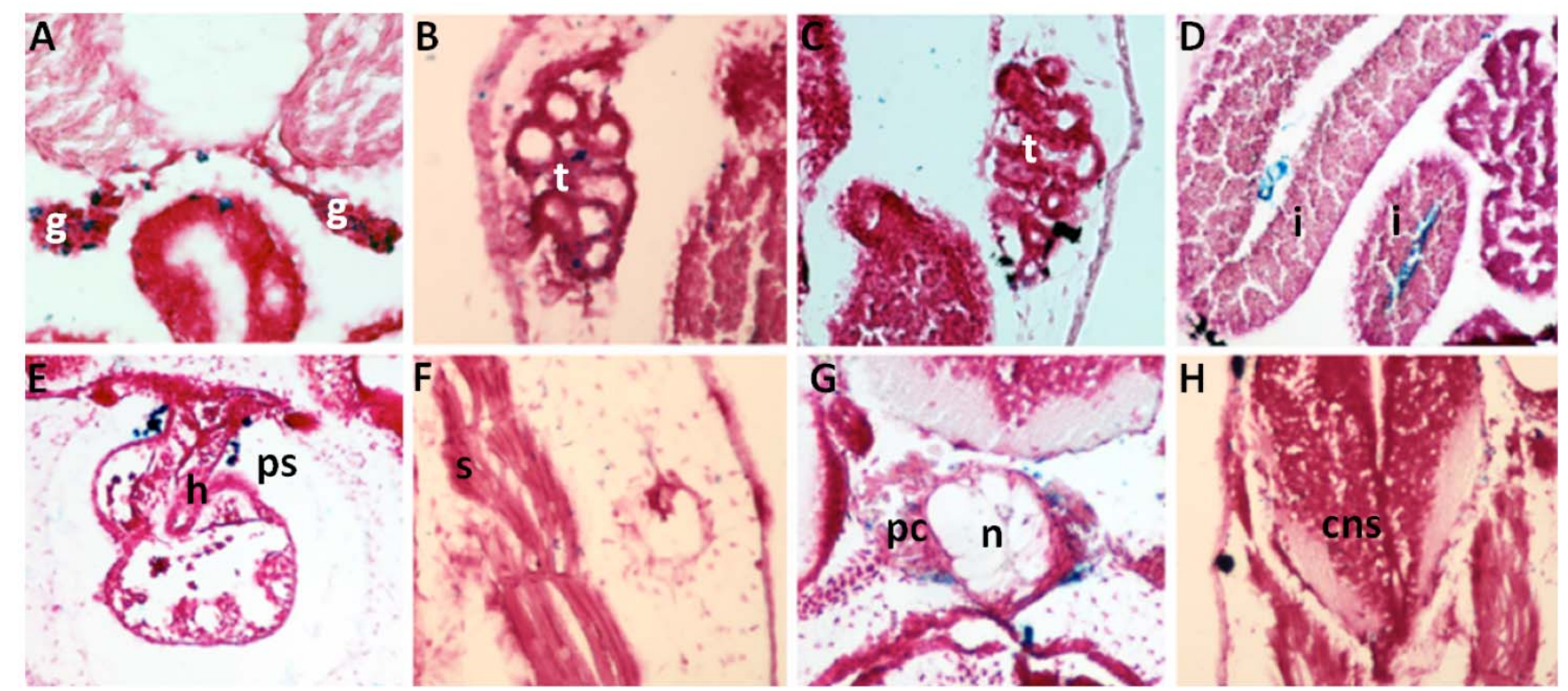

Figure 6: Iron staining of pronephric injected larvae sections (larvae fixed 72 hours after injection). h: heart; ps: pericardial sac; g: glomus; t: tubules; pe: peritoneum; on: optic nerve; pc: parachordal cartilage ; n: notochord; l: liver; pw: pharyngeal walls; cns: central nervous system; s: somites. The SPIONs are blue labeled, the sections are stained with pararosaniline and the black regions in $\mathrm{C}, \mathrm{D}$ and $\mathrm{H}$ are due to the natural larvae pigmentation.

in left pronephros (both glomus (Figure 6A) and tubules portion (Figure 6B)), gut (Figure 6D) and pericardial sac $(100 \%)$ (Figure 6E) in agreement with whole mount fluorescence data; the major concentration of SPIONs in the non-injected side was localized in the glomus and only at low level in the tubules portion of pronephros (100\%) (Figure 6C). Moreover SPIONs reached somites $(80 \%)$, parachordal cartilage $(100 \%)$ and the outer margin of the anterior central nervous system (100\%) (Figure 6F-H).

It is worth to mention that larvae not sacrificed for histology were monitored for 2 weeks. No mortality, malformations or interference with the normal development were registered (100\%).

\section{DISCUSSION AND CONCLUSION}

The toxicology of engineered nanomaterials is a relatively new and evolving field. Although the applications of nanoparticles (NPs) are increasing in many fields, concerns about their environmental and health impacts remain unresolved. The safety risk cannot be ignored as commercial applications of nanoparticles are accompanied by a lack of safety regulations and toxicology data. Various biological models have been proposed for toxicological assessments including in-vitro and in-vivo methodologies. In-vitro studies, based on cell cultures, are rapid, efficient, and low cost. However, results from these studies often provide an incomplete assessment of toxicity to the whole organism. Toxicology studies using in-vivo systems carry greater reliability, address the overall effect on the physiology and anatomy of the organism, and hence constitute a more relevant platform for translational clinical studies. The widely accepted small animal models are both costly and labour intensive; they require extensive animal care facilities and large numbers of animals for robust toxicity assessments; additionally, such studies raise ethical issues and resistance from the anti-vivisectionist lobby because of the large number of animals which have to be used for statistical significance. In recent years, a powerful alternative to small mammals has emerged with the use of aquatic in-vivo systems, which are now widely accepted and used for toxicological studies. Xenopus has been a well-established model for eco-toxicology over several decades and are in established use in drug development. Furthermore, the methods and protocols used for eco-toxicology can be easily adapted to nano-toxicological studies. For example, the Frog Embryo Teratogenesis AssayXenopus (FETAX) protocol is a well-established tool for evaluating embryotoxicity in amphibians and it has been used also for the evaluation of nanomaterials toxicity [18]. Interestingly, in this study the level of toxicity was found comparable to those described in the literature with other biological models, indicating that $\mathrm{TiO}_{2}$ is less toxic than $\mathrm{CuO}$ and $\mathrm{ZnO}$, in agreement with studies on other models, i.e., E. coli [19], yeast [20], human cell lines [21, 22] and zebrafish [23]. The study showed that, although transition metal oxide NPs do 
not cause mortality at concentrations up to $500 \mathrm{mg} / \mathrm{L}$, they affect embryo development, causing significant malformation rates, with the gut being the main target organ; moreover growth retardation and cephalic malformation were documented [18]. Xenopus laevis is thus as a powerful model system to study toxicity, not only in terms of survival, but also to investigate functional interactions with engineered nanomaterials, and for the diagnosis of early toxic stress.

Xenopus is a good model also for microinjection and microsurgical studies. Starting from the first divisions, the embryos have clearly distinguishable left (L) and right (R), dorsal (D) and ventral (V) blastomeres, which are very useful during NPs microinjections as the contralateral non-injected side provides an internal control. Most importantly, some organs are derived specifically from $\mathrm{D}$ and $\mathrm{V}$ blastomeres, providing the possibility to investigate the fate of the injected NPs. Additionally, Xenopus is a powerful tool for pluripotent cell assays in transplant experiments. Xenopus laevis "animal cap" cells are pluripotent cells situated around the animal (pigmented) pole of a blastula or very early gastrulastage embryo [24]. The animal cap cells can be removed from an embryo and cultured in-vitro exposed to the NPs, before transplantation to an untreated embryo.

We suggest that results obtained with this model have relevance to human health since the genes of $X$. laevis share a high degree of homology to the human genome and specific genes involved in developmental pathways are de-regulated in many human cancers. In particular many cellular, anatomical, and physiological characteristics are similar to those of humans and other vertebrates [25].
The advantage for this model for nanomedicine studies are summarised in Table 1.

There are reports on the use of Xenopus in imaging studies with NPs. Specifically, quantum dots (QDs) have been injected in Xenopus embryos for studying morphogenesis [26, 27], to label cell surface proteins such as $G_{A B A}$ surface receptors [28] or focaladhesion complexes [29] and to measure cellular and embryonic fluids movements, as exemplified by the in vivo measurement of the migration of Xenopus mesodermal cells $(1.62-2.18 \mu \mathrm{m} / \mathrm{min})$ reported by Stylianou and Skourides [27].

In the present study we used SPIONs and injected these NPs in heart and pronephros, two organs easily accessible by microinjection. In the only previous report in literature using Xenopus embryos [30], the MNPs were not injected; instead 5 weeks tadpoles were exposed to MNPs for 24 hours and, as expected, the MNPs accumulated in embryonic digestive tracts. In the present study, SPIONs were injected in Xenopus larva heart, following which, SPIONs were found throughout the whole embryo: instead of localizing in the injected organ, they were mainly targeted to eye, nervous system, gut and pronephros (Figures 2, 5). Similarly, when injected into the left pronephros, SPIONs were detected not only in pronephros structures, but also in CNS, heart, notochord, intestine and liver of embryos (Figures 3, 4, 6). The crucial finding is related to the results following injection of the left pronephros which confirmed diffusion of SPIONs to the non-injected side, as shown in Figure 3, where both glomus were labeled by SPIONs. Our results suggest an involvement of the blood stream in the dispersion and, in some cases, we could observe directly under the microscope the walk of SPIONs throughout the blood vessels. SPION spreading seems to stop at 72 96 hours after injection.

Table 1:

Advantages of the use of Xenopus embryos in nanomedicine

- Embryos offer the optimal size $(1.2 \mathrm{~mm})$, large enough for easy experimental manipulation and microsurgery

- Embryos develop externally

- Embryos are easy to obtain in large numbers, providing statistical power to the analysis and facilitating the collection of material for biochemical studies including proteomics

- Rapid development makes them ideal rapid assessment models for screening

- $\quad$ The small quantity needed to fully evaluate biological responses to a novel engineered nanoparticle is another advantage (compared to small mammalian models, which amounts several orders of magnitude greater)

- They constitute a valuable model for studying the effects of nanomaterials

- They are vertebrate with well-enough characterized transcriptomes, and have a high degree of homology to the human genome

- $\quad$ They are recognized as a valid model for studying human disease 
The diffusion of nanoparticles by the blood-flow has been already reported in Xenopus by Bacchetta et al. [18]. These authors observed that, following exposure after larva stage in Xenopus, the NMs accumulate mainly in digestive tract lumen (via oral route) and from the gut NMs diffuse in blood to reach liver and heart.

In conclusion we tested magnetic nanoparticles with a hydrodynamic size of about $200 \mathrm{~nm}$. When these relatively large nanoparticles are injected in 37 stage $X$. laevis larvae, they spread over the whole embryo probably via the blood stream diffusing in many organs. $X$. laevis represents thus a powerful model to study the fate of systemically administered nanoparticles.

\section{ACKNOWLEDGEMENTS}

This work has been partially supported by MBIAD project (Role of multilayer nanoencapsulation, antiinflammatory nanostructures, and selective nanoparticle-guided homing in human islet transplantation for the treatment of type 1 diabetes) founded by Fondazione Cassa di Risparmio di Pisa.

\section{REFERENCES}

[1] Fako VE, Furgeson DY. Zebrafish as a correlative and predictive model for assessing biomaterial nanotoxicity. Adv Drug Deliv Rev 2009; 61: 478-86. http://dx.doi.org/10.1016/j.addr.2009.03.008

[2] Tseng CL, Peng CL, Huang JY, Chen JC, Lin FH. Gelatin nanoparticles as gene carriers for transgenic chicken applications. J Biomater Appl 2012; DOI: $0.1177 / 0885328211434089$.

[3] Nieuwkoop PD, Faber J. Normal Table of Development of Xenopus laevis (Daudin). North- Holland, Amsterdam: Elsevier 1967.

[4] Gao JH, Gu HW, Xu B. Multifunctional Magnetic Nanoparticles: Design, Synthesis, and Biomedical Applications. Accounts Chem Res 2009; 42: 1097-107. http://dx.doi.org/10.1021/ar9000026

[5] Indira TK, Lakshimi PK. Magnetic Nanoparticles: A Review. Int J Pharm Sci Nanotech 2010; 3: 8.

[6] Pankhurst QA, Connolly J, Jones SK, Dobson J. Applications of magnetic nanoparticles in biomedicine. J Physics D-Appl Phys 2003; 36: R167-R81.

[7] Getzlaff M. Fundamentals of Magnetism, Springer, New York, NY, USA 2008.

[8] Bulte JWM. In vivo MRI cell tracking: clinical studies. Am J Roentgenol 2009; 193: 314-25.

http://dx.doi.org/10.2214/AJR.09.3107

[9] Kim HS, Oh SY, Joo HJ, Son K-R, Song I-C, Moon WK. The effects of clinically used MRI contrast agents on the biological properties of human mesenchymal stem cells. NMR Biomed 2010; 23: 514-22. http://dx.doi.org/10.1002/nbm.1487

[10] Wu S, Zhang L, Zhong J, Zhang Z. Dual contrast magnetic resonance imaging tracking of iron-labeled cells in vivo. Cytotherapy 2010; 12: 859-69.

http://dx.doi.org/10.3109/14653241003587652

[11] Jain TK, Richey J, Strand M, Leslie-Pelecky DL, Flask CA, Labhasetwar V. Magnetic nanoparticles with dual functional properties: Drug delivery and magnetic resonance imaging. Biomaterials 2008; 29: 4012-21.

http://dx.doi.org/10.1016/j.biomaterials.2008.07.004

[12] Wahajuddin SA. Superparamagnetic iron oxide nanoparticles: magnetic nanoplatforms as drug carriers. Int $\mathrm{J}$ Nanomed 2012; 7: 3445-71.

http://dx.doi.org/10.2147/IJN.S30320

[13] Berensmeier S. Magnetic particles for the separation and purification of nucleic acids. Appl Microbiol Biotechnol 2006; 73: 495-504.

http://dx.doi.org/10.1007/s00253-006-0675-0

[14] Scherer F, Anton M, Schillinger U, Henke J, Bergemann C Krüger A, Gänsbacher B, Plank C. Magnetofection: enhancing and targeting gene delivery by magnetic force in vitro and in vivo. Gene Therapy 2002; 9: 102-109. http://dx.doi.org/10.1038/sj.gt.3301624

[15] Jordan KM. Efficacy and safety of intratumoral thermotherapy using magnetic iron-oxide nanoparticles combined with external beam radiotherapy on patients with recurrent glioblastoma multiforme. J Neurooncol 2011; 103: 317-24.

http://dx.doi.org/10.1007/s11060-010-0389-0

[16] Newport J, Kirschner M. A major developmental transition in early Xenopus embryos. II. Control of the onset of transcription. Cell 1982; 30: 687-96. http://dx.doi.org/10.1016/0092-8674(82)90273-2

[17] Sive HL, Grainger RM, Harland RM. Early Development of Xenopus laevis: A Laboratory Manual, Cold Spring Harbor, NY, Cold Spring Harbor Laboratory Press 2000.

[18] Bacchetta $R$, Santo N, Fascio U, Moschini E, Freddi S, Chirico G, et al. Nano-sized $\mathrm{CuO}, \mathrm{TiO}_{2}$ and $\mathrm{ZnO}$ affect Xenopus laevis development. Nanotoxicology 2012; 6: 38198.

http://dx.doi.org/10.3109/17435390.2011.579634

[19] Hu X, Cook S, Wang P, Hwang HM. In vitro evaluation of cytotoxicity of engineered metal oxide nanoparticles. Sci Total Environ 2009; 407: 3070-2. http://dx.doi.org/10.1016/j.scitotenv.2009.01.033

[20] Kasemets K, Ivask A, Dubourguier HC, Kahru A. Toxicity of nanoparticles of $\mathrm{ZnO}, \mathrm{CuO}$ and $\mathrm{TiO}_{2}$ to yeast Saccharomyces cerevisiae. Toxicol In Vitro 2009; 23: 111622.

http://dx.doi.org/10.1016/j.tiv.2009.05.015

[21] Lai JC, Lai MB, Jandhyam S, Dukhande VV, Bhushan A, Daniels $C K$, et al. Exposure to titanium dioxide and other metallic oxide nanoparticles induces cytotoxicity on human neural cells and fibroblasts. Int J Nanomed 2008; 3: 533-45.

[22] Karlsson HL, Cronholm P, Gustafsson J, Moller L. Copper oxide nanoparticles are highly toxic: A comparison between metal oxide nanoparticles and carbon nanotubes. Chem Res Toxicol 2008; 21: 1726-32. http://dx.doi.org/10.1021/tx800064j

[23] Lin S, Zhao Y, Xia T, Meng H, Ji Z, Liu R, et al. High content screening in zebrafish speeds up hazard ranking of transition metal oxide nanoparticles. ACS Nano 2011; 5: 7284-95. http://dx.doi.org/10.1021/nn202116p

[24] Lan L, Vitobello A, Bertacchi M, Cremisi F, Vignali R, Andreazzoli $M$, et al. Noggin elicits retinal fate in Xenopus animal cap embryonic stem cells. Stem Cells 2009; 27: 214652. http://dx.doi.org/10.1002/stem.167

[25] Pearl EJ, Grainger RM, Guille M, Horb ME. Development of Xenopus Resource Centers: The National Xenopus Resource and the European Xenopus Resource Center. Genesis 2012; 50: 155-63. http://dx.doi.org/10.1002/dvg.22013

[26] Dubertret B, Skourides P, Norris DJ, Noireaux V, Brivanlou $\mathrm{AH}$, Libchaber $\mathrm{A}$. In vivo imaging of quantum dots 
encapsulated in phospholipid micelles. Science 2002; 98: 1759-62.

http://dx.doi.org/10.1126/science.1077194

[27] Stylianou P, Skourides PA. Imaging morphogenesis, in Xenopus with Quantum Dot Nanocrystals. Mech Dev 2009; 126: 828-41.

http://dx.doi.org/10.1016/j.mod.2009.07.008

[28] Gussin HA, Tomlinson ID, Little DM, Warnement MR, Qian $\mathrm{H}$, Rosenthal SJ, et al. Binding of muscimol-conjugated quantum dots to GABAC receptors. J Am Chem Soc 2006; 128: 15701-13.

http://dx.doi.org/10.1021/ja064324k
[29] Charalambous A, Antoniades I, Christodoulou N, Skourides PA. Split-Inteins for Simultaneous, site-specific conjugation of Quantum Dots to multiple protein targets in vivo. J Nanobiotechnol 2011; 9: 37.

http://dx.doi.org/10.1186/1477-3155-9-37

[30] Oldenburg A, Toublan F, Suslick K, Wei A, Boppart S. Magnetomotive contrast for in vivo optical coherence tomography. Opt Express 2005; 13: 6597-14. http://dx.doi.org/10.1364/OPEX.13.006597

Received on 23-04-2013

Accepted on 13-05-2013

Published on 22-08-2013

DOI: http://dx.doi.org/10.12974/2311-8792.2013.01.01.2

(C) 2013 Giannaccini et al.; Licensee Savvy Science Publisher.

This is an open access article licensed under the terms of the Creative Commons Attribution Non-Commercial License (http://creativecommons.org/licenses/by-nc/3.0/) which permits unrestricted, non-commercial use, distribution and reproduction in any medium, provided the work is properly cited. 